\title{
Double Grating Design of 3D Phase Matched Waveguide for Second Harmonic $\chi^{(2)}$ Process
}

\author{
*Alessandro Massaro, ${ }^{*}$ Roberto Cingolani, ${ }^{*}$ Massimo De Vittorio, and ${ }^{*}, \mathrm{~A}$ Adriana Passaseo. \\ "National Nanotechnology Laboratory of CNR-INFM , Distretto Tecnologico-ISUFI, Università del \\ Salento,Via Arnesano, 73100 Lecce- Italy. \\ ${ }^{A}$ Permanent address: IMM-CNR sezione Lecce, University Campus, Lecce-Monteroni 73100, Italy.
}

\begin{abstract}
Theoretical analysis on second harmonic (SH) generation with phase matched grating in waveguide is presented from the viewpoint of device design. Usually high intensity sources are necessary in order to observe a SH in a $\chi^{(2)}$ nonlinear structure. For this purpose, the novel proposed design takes into account a double grating effect which enhances the guided SH signal along the waveguide. In the presented structure two grating are considered: the first grating, considered at the interface between air and core, is designed in order to obtain an efficient SH conversion process by considering the quasi phase matching (QPM) condition; the second grating, placed at the interface between the core and the substrate region, increases the SH power along the propagation direction through the coupling with the substrate modes generated by the diffraction effect. The novelty of this work is in the combined effect of the two gratings. The grating lengths and periods are designed by considering the nonlinear coupled mode theory with the effective dielectric constant (EDC) assumption. The analysis includes three dimensional (3D) cases where phase matching is involved, in particular the model is applied to a GaAs/AlGAs waveguides with fundamental wavelength at $\lambda_{\mathrm{FU}}=1.55 \mu \mathrm{m}$ and $\mathrm{SH}$ signal at $\lambda_{\mathrm{SH}}=0.775 \mu \mathrm{m}$.
\end{abstract}

Keywords: Nonlinear Waveguides, Second Harmonic Generation Process, Grating Diffraction, Scalar Hertzian Potential.

\section{INTRODUCTION}

Studies of $\chi^{(2)}$ processes and the quasi-phase matching (QPM) technique promote research works on nonlinear optic (NLO) devices for ultrafast signal processing based on second-order nonlinearity. After the development of highefficiency QPM- second harmonic generation (SHG) devices using ferroelectric crystal waveguides, the research was directed to implementation of a compact and efficient coherent light source by combining the SHG device with a semiconductor laser. Moreover a strong motivation of research on NLO devices has been the need for the development of all-optical wavelength converters for dense wavelength division multiplexing (DWDM) optical communication systems. In particular the second harmonic generation in $\chi^{(2)}$ nonlinear process represents a good solution for DWDM applications in telecommunication systems. But this process requires high intensity input power and large interaction lengths for a good second harmonic $(\mathrm{SH})$ conversion. For this reasons the introduction of tailored gratings which enhance the SH field is important. The novel design proposed in this work takes into account the multiple grating effects which enhance the guided SH signal along the symmetrical waveguide shown in Fig. 1. In the presented structure two gratings are considered: the first grating (grating 1 of Fig. 1), considered at one interface between core and substrate, is designed by QPM condition (the QPM technique [1]-[4] is a practical method which increases the second-harmonic power by effectively reducing the phase mismatch between the fundamental and the SH modes); moreover a second grating (grating 2 of Fig. 1), placed at second the interface between the core and the substrate region, increases the fundamental power through the coupling between the substrate modes (radiation modes) [5]-[7] and the fundamental guided mode. The substrate modes are excited through the diffraction effect of the grating 2 of Fig. 1 which supplies the energy to both the propagating fields through the coupled energy of the radiation modes: the grating 2 enhances the fundamental mode which will transfer the energy to the SH propagating mode through grating 1 . The coupling effect is analyzed by combining the coupled mode equations systems related to the two gratings. The originality of this work is in the combined effect of the two gratings applied to a symmetrical GaAs/AlGaAs waveguide typically used in NLO

Nonlinear Optics and Applications III, edited by Mario Bertolotti, Proc. of SPIE Vol. 7354, 73540V · (C) 2009 SPIE · CCC code: 0277-786X/09/\$18 · doi: 10.1117/12.820443 
devices [1]-[3]. In particular we analyze the enhancement of a SH signal $\left(\lambda_{\mathrm{SH}}=0.775 \mu \mathrm{m}\right)$ generated by a codirectional fundamental mode $\left(\lambda_{\mathrm{FU}}=1.55 \mu \mathrm{m}\right)$ though the nonlinear effect of the core. The goal is to find the optimum geometrical configuration which overcomes the problems associated to the total waveguide length such as reflections and losses, by providing a good SH field conversion. With the introduction of the multiple grating configuration it is possible to decrease the total waveguide length by obtaining a good conversion efficiency according to real input powers.

We resume the analysis of this work in the following steps: i) we study the coupled mode equations systems by combining the effect of each grating; ii) we provide the design criteria steps of the periodic structure; iii) finally we analyze the grating effects in a $3 \mathrm{D}$ ridge waveguides.

\subsection{Analytical Model}

In our analysis two coupled equations system are considered. The first set of coupled equations is related to grating 1 of Fig. 1. The function of this grating is to couple the fundamental field with the SH field. In particular by indicating with $A^{\omega}$ the amplitude of the pump fundamental field and with $A^{2 \omega}$ the amplitude of the SH wave we obtain [8],[9]

$$
\left\{\begin{array}{l}
\frac{d A^{\omega}(z)}{d z}+j\left(2 k_{L}^{\omega} \cos K z\right) A^{\omega}(z) \\
=-j\left[k_{N L} \exp (j 2 \delta z)\right]^{*}\left[A^{\omega}(z)\right]^{*} A^{2 \omega}(z) \\
\frac{d A^{2 \omega}(z)}{d z}+j\left(2 k_{L}^{2 \omega} \cos K z\right) A^{\omega}(z) \\
=-j k_{N L} \exp (j 2 \delta z)\left[A^{\omega}(z)\right]^{2}
\end{array}\right.
$$

with

$$
\begin{aligned}
& k_{L}^{\omega}=\frac{\omega \varepsilon_{0}}{4} \int\left[E_{y}^{\omega}(x)\right]^{*} \Delta \varepsilon E_{y}^{\omega}(x) d x \\
& k_{L}^{2 \omega}=\frac{2 \omega \varepsilon_{0}}{4} \int\left[E_{y}^{2 \omega}(x)\right]^{*} \Delta \varepsilon E_{y}^{2 \omega}(x) d x \\
& k_{N L}=\frac{2 \omega \varepsilon_{0}}{4} \int\left[E_{y}^{2 \omega}(x)\right]^{*} \tilde{\chi}_{N L}\left[E_{y}^{\omega}(x)\right]^{2} d x
\end{aligned}
$$

where

$$
2 \delta=\beta^{2 \omega}-\left(2 \beta^{\omega}+2 \pi / \Lambda_{1}\right)
$$

is the phase mismatch between the fundamental and SH field,

$$
\Delta \varepsilon=\left(\varepsilon_{1}-\varepsilon_{2}\right) \frac{\sin (a \pi)}{\pi} \exp \left[-j z 2 \pi / \Lambda_{1}\right]
$$

is the first term of the permittivity Fourier series, and finally

$$
\tilde{\chi}_{N L}=\left(\chi_{1}^{(2)}-\chi_{2}^{(2)}\right) \frac{\sin (a \pi)}{\pi} \exp \left[-j z 2 \pi / \Lambda_{1}\right]
$$


is the first term of the nonlinear coefficient Fourier series. The parameter $a$ denotes the duty ratio of the period, and the term $\left(\chi_{1}{ }^{(2)}-\chi_{2}{ }^{(2)}\right)$ indicates the periodically switched nonlinearity (PSN) [1] related to a $\mathrm{GaAs}_{\mathrm{As}} / \mathrm{Al}_{0.4} \mathrm{Ga}_{0.6} \mathrm{As}$ structure. The grating 2 of Fig. 1 enhances the low pump fundamental field coupled into the waveguide. In this case coupling between the fundamental field and the scattered fields (generated by grating 2) is considered. In particular the equations to solve are the following [10]

$$
\left\{\begin{array}{c}
\frac{d A^{\omega}(z)}{d z}=-j \sum_{i} \int a_{\gamma i}^{ \pm}(z) k_{\gamma i, A^{\omega}}^{*} \exp \left[-j\left(\beta^{ \pm}-\left(\beta^{\omega}-2 \pi / \Lambda_{2}\right) z\right] d \beta^{ \pm}\right. \\
\frac{d a_{\gamma i}^{ \pm}(z)}{d z}=-j A^{\omega}(z) k_{\gamma i, A^{\omega}}^{*} \exp \left[-j\left(\beta^{ \pm}-\left(\beta^{\omega}-2 \pi / \Lambda_{2}\right) z\right]\right.
\end{array}\right.
$$

with the coupling coefficient defined as

$$
k_{\gamma i, A^{\omega}}=k_{S}^{\omega}=\int E_{y}^{\omega}(x) \Delta \varepsilon E_{y}^{S}(x) d x
$$

The mode amplitudes $a_{\gamma \mathrm{i}}{ }^{ \pm}$represent the radiation modes generated by the diffraction effect of the grating 2 [6],[7]. The coupling coefficients (2), (3), (4), and (9) are obtained by the guided electric field [5].

$$
\begin{aligned}
& E_{y}^{\omega}=a^{\omega} \psi^{\omega}(x) e^{-j \beta^{\omega} z} \\
& E_{y}^{2 \omega}=a^{2 \omega} \psi^{2 \omega}(x) e^{-j \beta^{2 \omega} z}
\end{aligned}
$$

where

$$
\begin{gathered}
\psi^{\omega, 2 \omega}(x)=\left\{\begin{array}{cc}
\cos \left(\sigma^{\omega, 2 \omega} x\right) & \text { core } \\
\cos \left(\sigma^{\omega, 2 \omega} d / 2\right) \exp \left[-\Delta^{\omega, 2 \omega}(x-d / 2)\right] & \text { substrate }
\end{array}\right. \\
a^{\omega, 2 \omega}=\sqrt{\frac{d}{1+\frac{1}{\Delta^{\omega, 2 \omega}}}}
\end{gathered}
$$

and the parameters $\sigma^{\omega, 2 \omega}$ and $\Delta^{\omega, 2 \omega}$ (propagation constants in the substrate and core region, respectively) are defined by the following equations

$$
\begin{aligned}
& \Delta^{\omega, 2 \omega}=\left(n_{2}^{2}\left(k^{\omega, 2 \omega}\right)^{2}-\left(\beta^{\omega, 2 \omega}\right)^{2}\right)^{1 / 2} \\
& \sigma^{\omega, 2 \omega}=\left(n_{1}^{2}\left(k^{\omega, 2 \omega}\right)^{2}-\left(\beta^{\omega, 2 \omega}\right)^{2}\right)^{1 / 2}
\end{aligned}
$$

and by the scattered field [6],[7]

$$
\begin{array}{r}
E_{y}^{S}=\frac{t}{2} A_{i} \sigma^{\omega, 2 \omega}\left(\Delta^{\omega, 2 \omega}-\sigma^{\omega, 2 \omega}\right)\left[\frac{\exp \left[-j\left(\sigma^{(-)} x-\beta^{(-)} z\right)\right]}{\sigma^{(-)}+\Delta^{(-)}}-\frac{\exp \left[j\left(\sigma^{(+)} x-\beta^{(+)} z\right)\right]}{\sigma^{(+)}+\Delta^{(+)}}\right] \\
\text {core region }
\end{array}
$$




$$
E_{y}^{S}=\frac{t}{2} A_{i} \sigma^{\omega, 2 \omega}\left(\Delta^{\omega, 2 \omega}-\sigma^{\omega, 2 \omega}\right)\left[\frac{\exp \left[-j\left(\Delta^{(-)} x+\beta^{(-)} z\right)\right]}{\sigma^{(-)}+\Delta^{(-)}}-\frac{\exp \left[-j\left(\Delta^{(+)} x+\beta^{(+)} z\right)\right]}{\sigma^{(+)}+\Delta^{(+)}}\right]
$$

with the definitions [7]

$$
\begin{aligned}
& \beta^{( \pm)}=\beta^{\omega, 2 \omega} \pm \frac{2 \pi}{\Lambda_{2}} \\
& \sigma^{( \pm)}=\left(n_{1}^{2}\left(k^{\omega, 2 \omega}\right)^{2}-\left(\beta^{( \pm)}\right)^{2}\right)^{1 / 2} \\
& \Delta^{( \pm)}=\left(n_{2}^{2}\left(k^{\omega, 2 \omega}\right)^{2}-\left(\beta^{( \pm)}\right)^{2}\right)^{1 / 2}
\end{aligned}
$$

where $\beta^{( \pm)}$indicates the propagation constant of the scattered waves. The scattered field is obtained through sinusoidal approximation of the dielectric profile of grating 2 [6],[7].

\subsection{Design and Results}

We assume that the fundamental pump source $\left(\lambda_{\mathrm{FU}}=1.55 \mu \mathrm{m}\right)$ is a polarized TE field. The GaAs core $\left(\mathrm{n}_{1}\left(\lambda_{\mathrm{FU}}=1.55\right.\right.$ $\mu \mathrm{m})=3.374)$ and $\left.\mathrm{n}_{1}\left(\lambda_{\mathrm{SH}}=0.755 \mu \mathrm{m}\right)=3.691\right)$ is characterized by a thickness $\mathrm{d}=0.22 \mu \mathrm{m}$ and supports only a TE fundamental mode at $\lambda_{\mathrm{FU}}=1.55 \mu \mathrm{m}$ and only a SH TE mode at $\lambda_{\mathrm{FU}}=0.775 \mu \mathrm{m}$. In this way all the TE power is matched with the two propagating modes and the modal dispersion is low. In order to minimize the reflections along the $z$ propagating direction and to conserve the single mode condition, the parameters $\mathrm{h}$ and $\mathrm{t}$ of Fig. 1 are fixed to the low value of $0.025 \mu \mathrm{m}$. The substrate material is $\mathrm{Al}_{0.4} \mathrm{Ga}_{0.6} \mathrm{As}\left(\mathrm{n}_{2}\left(\lambda_{\mathrm{FU}}=1.55 \mu \mathrm{m}\right)=3.2\right.$ and $\left.\mathrm{n}_{2}\left(\lambda_{\mathrm{SH}}=0.755 \mu \mathrm{m}\right)=3.4\right)$. The GaAs core, combined with the $\mathrm{Al}_{0.4} \mathrm{Ga}_{0.6} \mathrm{As}$ substrate, characterizes the periodically switched nonlinearity (PSN) [1] of the periodic waveguide. For the PSN effect to occur, a medium is required in which susceptibility coefficient $\chi^{(2)}$ is modulated periodically along the direction of light propagation. We observe that in a generic case $\chi^{(2)}$ is a tensor, however most semiconductor, which crystallize in zinc-blende structures, have a symmetry and their second-order susceptibility has a single nonzero independent component (for GaAs at the transparency region below the optical energy gap $\left.\chi_{x, y, z}{ }^{(2)}=200 \mathrm{pm} / \mathrm{V}[4]\right)$. The grating 1 couples the fundamental mode with the SH one. The period $\Lambda_{1}=2.31 * 10^{-6} \mu \mathrm{m}$ is fixed in order to satisfy the QPM condition (phase mismatch $\delta=0$ ). The duty ratio $a$ is assumed equal to $1 / 2$. The fundamental $A^{\omega}$ and $\mathrm{SH} A^{2 \omega}$ modes generated by this QPM period $\Lambda_{1}$ are reported in Fig. 2 . The second harmonic conversion efficiency $\eta=\left|A^{2 \omega}(\mathrm{z}=\mathrm{L})\right|^{2} /\left|A^{\omega}(0)^{2}\right|[8]$ changes for a 3D structure with the geometrical parameter $W$ which represents the width of the first grating: in particular, as reported in Fig. 3 (a), an high efficiency is obtained for large widths. Concerning the analysis of the second grating, we first considers the two dimensional (2D) model: this model takes into account the enhancement of the fundamental power by considering the radiation mode coupling of the grating 2 of Fig. 1. The grating 2 is designed in order to enhance the fundamental power inside the waveguide through the coupling with the scattered field generated by the same grating. Consecutively, the SH power will increase with the coupling condition (4). In this case the set of equations to solve simultaneously are (1) and (8). We observe that the period $\Lambda_{2}$ provides a constructive interference given by the phase matching condition (17) between the incident and the scattered field. We choose a strong coupling condition by analyzing the coupling coefficient versus the period $\Lambda_{2}$ and versus the $z$-direction. Figure 3 (b) shows that a strong coupling coefficient (9), is obtained with $\Lambda_{2}=0.95^{*} 10^{-6}$. The plot of Fig. 3 (b) is obtained by evaluating the scattered total field $E_{y}{ }^{S}$ of equation (15) as superposition of scattered fields generated by the fundamental and SH modes. The analytical solution of the SH conversion efficiency $\eta=\left|A^{2 \omega}(z=L)\right|^{2} /\left|A^{\omega}(0)\right|^{2}[8]$ for a two dimensional (2D) device is reported in Fig. 4 (a) which shows the effect of only the grating 1 , and the combined effects of grating 1 and grating 2. Figure 4 (a) highlights the importance of the radiation coupling of grating 2 for an high $\eta$. The input power used during the calculus is $\mathrm{P}_{0}=100 \mathrm{~mW}$. 


\section{3D Ridge Waveguide}

The analytical approach of the coupling mode theory provides an alternative design approach for 3D structures: the length of the step discontinuities (total grating lengths) can be chosen in order to obtain a maximum SH converted signal at the output of the grating. As application of this analytical model we analyze a typical optical 3D periodic waveguide commonly used in multiplexing and filtering applications, such as the ridge waveguide. The presented ridge waveguide is characterized by GaAs core on $\mathrm{Al}_{0,7} \mathrm{Ga}_{0,3} \mathrm{As}$ substrate at working wavelength of $\lambda_{0}=1.55 \mu \mathrm{m}$. This $3 \mathrm{D}$ waveguide is analyzed by the effective dielectric constant (EDC) [5] method. Moreover, the coupling mode theory provides the longitudinal position where the coupling between the fundamental mode and the generated SH mode is characterized by an high intensity. As reported in Fig. 4 (b) a good SH conversion efficiency is obtained by considering a double grating design: the first QPM grating with period $\Lambda_{1}=2.31 \mu \mathrm{m}$ is placed between the core and the air cladding interface (see inset of Fig. 4 (b)), moreover, the second grating, placed between the core/substrate interface satisfies the condition (17) by enhancing the SH signal. The designed ridge is characterized by $\mathrm{S}=1 \mu \mathrm{m}, \mathrm{D}=0.22 \mu \mathrm{m}, \mathrm{d}=0.195 \mu \mathrm{m}\left(\mathrm{L}_{\mathrm{c}}=\mathrm{L}_{\mathrm{s}}=\Lambda_{1} / 2\right)$, $\chi^{(2)}(\mathrm{GaAs})=200 \mathrm{pm} / \mathrm{V}, \mathrm{n}_{1}=\mathrm{n}_{1}(\mathrm{GaAs}-\mathrm{core}), \mathrm{n}_{2}=\mathrm{n}_{2}$ (AlGaAs-substrate), $\mathrm{n}_{3}$ (air-cladding) $=1$.

\section{Conclusion}

We presented in this work a theoretical model which provides the second harmonic conversion efficiency in a nonlinear waveguide with discontinuous core-substrate and core-cladding interfaces. The model provides design criteria for second harmonic enhancement through the use of two gratings. The optimization of the SH enhancement is obtained through the radiation modes coupling. The design approach is applied to $3 \mathrm{D}$ optical waveguides such as ridge waveguides and can be extend to $3 \mathrm{D}$ photonic crystals. 
Figures and captions.

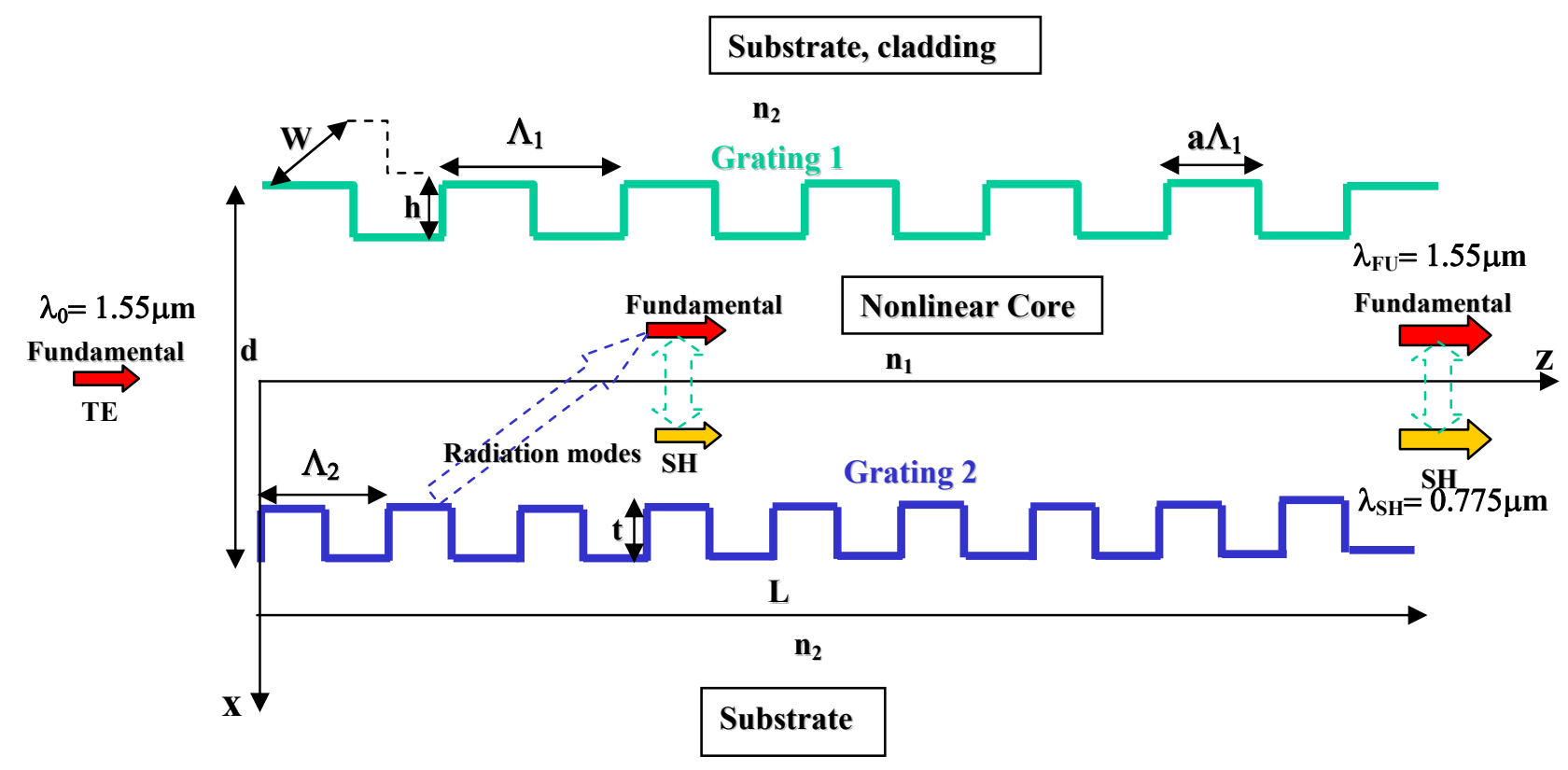

Fig. 1: Double grating design: second harmonic enhancement in a symmetrical waveguide with nonlinear core. The width $\mathrm{W}$ represents the 3D model.
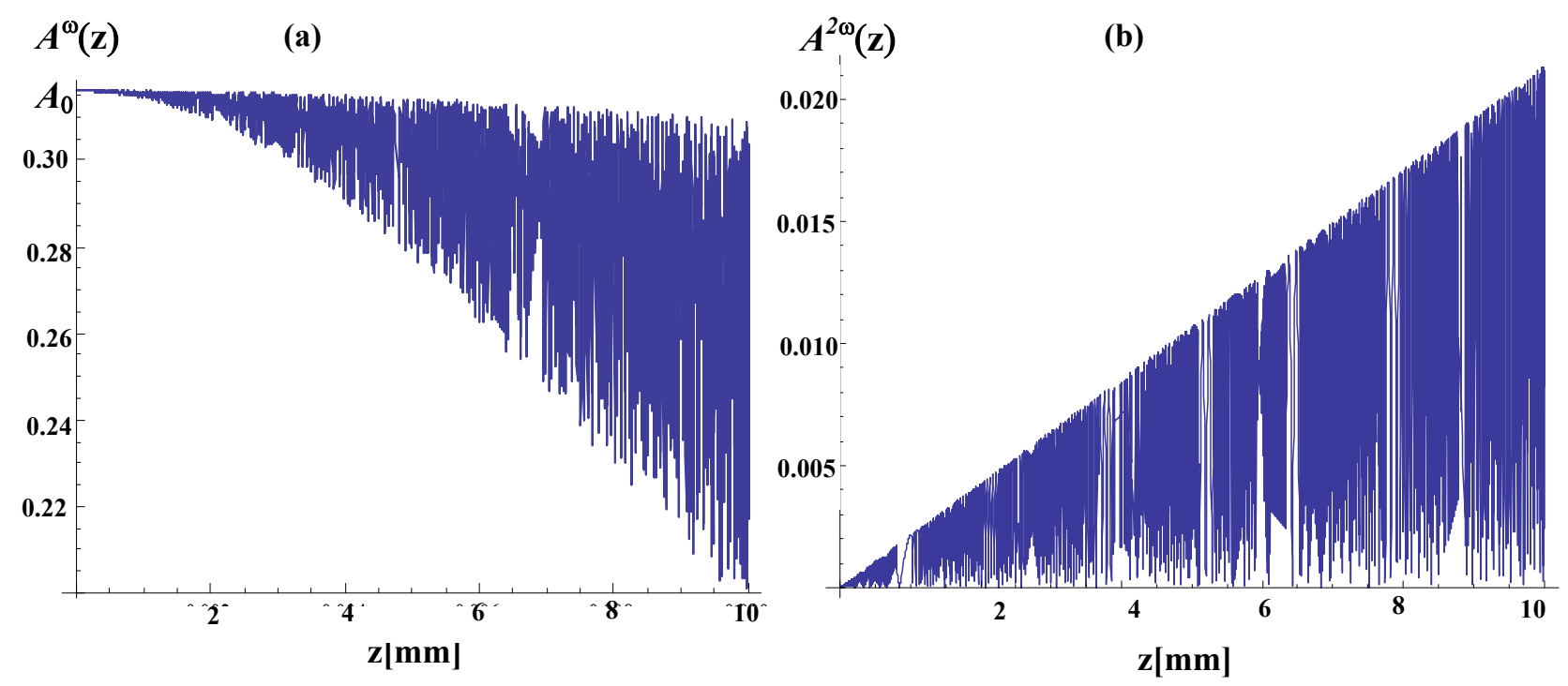

Fig. 2: Quasi phase matching condition: (a) fundamental field $A^{\omega}$ and (b) second harmonic field $A^{2 \omega}$ versus $z$ generated by the grating 1 of Fig. 1. 
(a)

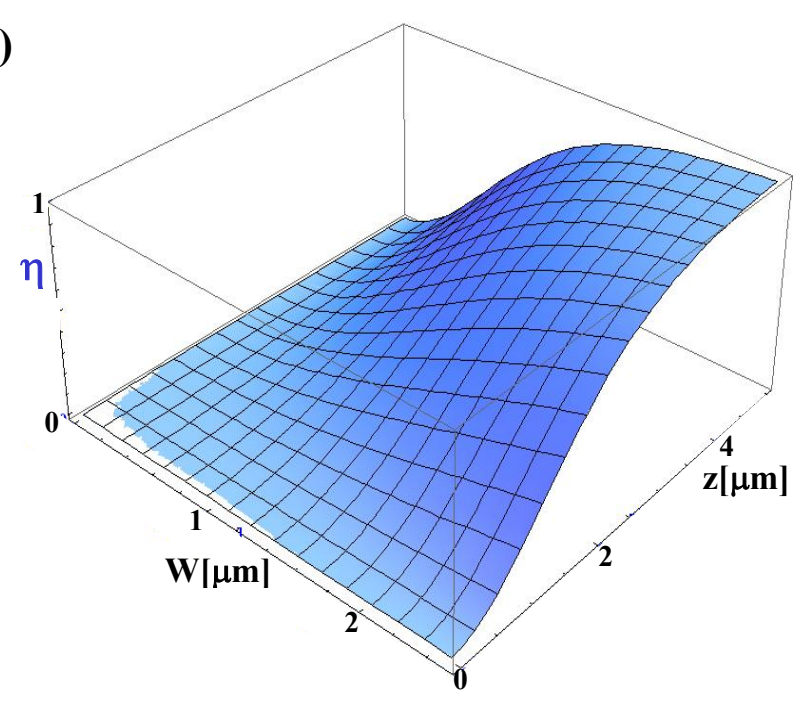

(b)

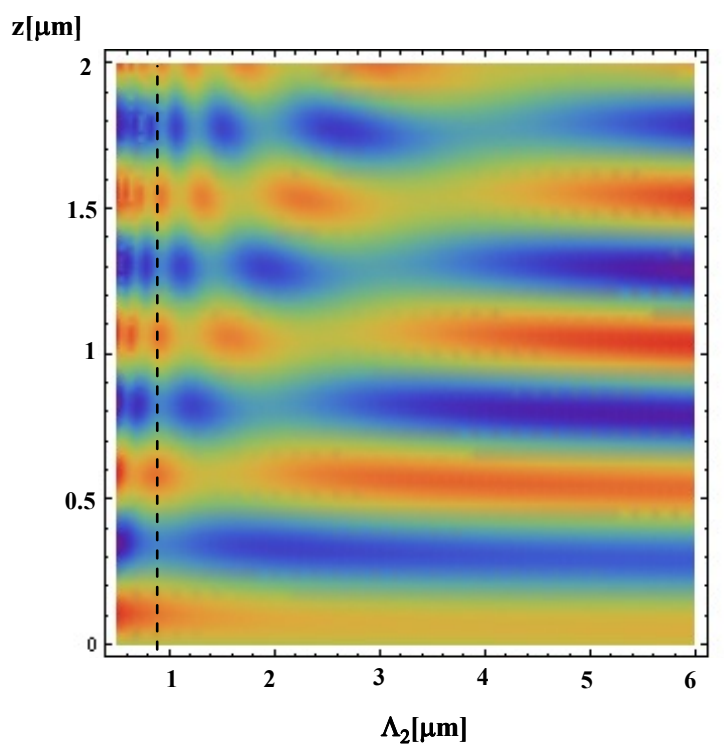

Fig. 3: (a) Second harmonic conversion efficiency versus $z$ and W. (b) Coupling coefficient of equation (9) versus $z$ and $\Lambda_{2}$ : a strong coupling is obtained with $\Lambda_{2}=0.95 \mu \mathrm{m}$.
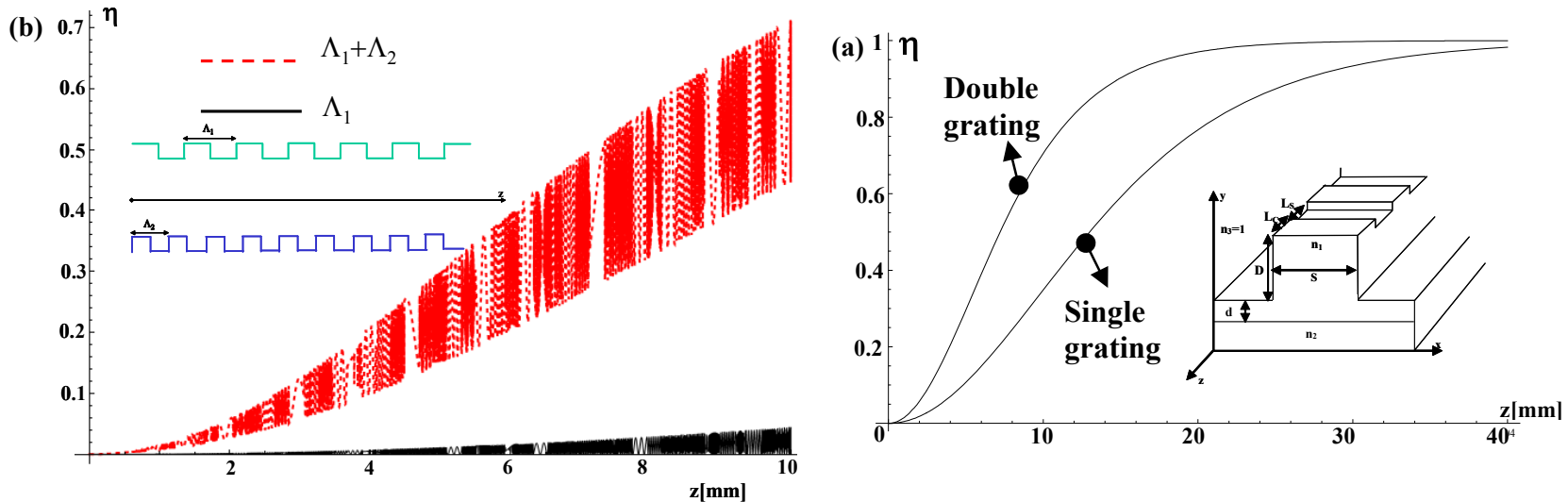

Fig. 4 (a) Symmetrical waveguide with double grating: second harmonic conversion efficiency versus $z$. (b) Second harmonic conversion efficiency versus $z$ in a ridge waveguide: single grating refers to the QPM grating with period $\Lambda_{1}$, and double grating considers a ridge waveguide with QPM grating (with period $\Lambda_{1}$ and placed at the core/cladding interface ) and with a second grating (with period $\Lambda_{2}$ and placed at the core/substrate interface) as indicated in the longitudinal section of Fig. 1. In this case the cladding is air and the substrate is AlGaAs. 


\section{REFERENCES}

1. E. U. Rafailov, P. L. Alvarez, C. T. A. Brown, W. Sibbett, R. M. De la Rue, P. Millar, D. A. Yanson, J. S. Roberts, and P. A. Houston, "Second-harmonic generation from a first-order quasi-phase-matched GaAs/AlGaAs waveguide crystal,” Opt. Lett. 26 (24), 1984-1986 (2001).

X. Yu, L. Scaccabarozzi, J. S. Harris, P. S. Kuo, and M. M. Fejer, "Efficient continuous wave second harmonic generation pumped at $1.55 \mu \mathrm{m}$ in quasi-phase-matched AlGaAs waveguides," Opt. Express 13 (26), 10742-10748 (2005).

3. X. Yu, L. Scaccabarozzi, A. C. Lin, M. M. Fejer, and J. S. Harris, "Growth of GaAs with orientation-patterned structures for nonlinear optics," J. of Crystal Growth 301, 163-167 (2007).

4. A. Massaro, V. Tasco, M. T. Todaro, R. Cingolani, M. De Vittorio, and A. Passaseo, "Scalar time domain modeling and coupling of second harmonic generation process in GaAs discontinuous optical waveguide," Opt. Express 16 (19), 14496-14511 (2008).

5. T. Rozzi and M. Mongiardo, Open Electromagnetic Waveguides, (IEE Electromagnetic Waves Series 43, London 1997).

6. D. Marcuse, Theory of Dielectric Optical Waveguides, (Academic Press, New York 1974).

7. D. Marcuse, "Hollow dielectric waveguides for distributed feedback lasers," IEEE J. Quantum Electron. 26 (7), 1265-1276 (1972).

8. T. Suhara, and M. Fujimura, Waveguide Nonlinear-Optic Devices, (Berlin: Springer, 2003).

9. T. Suhara, and H. Nishihara, "Theoretical analysis of waveguide second-harmonic generation phase matched with uniform and chirped grating," IEEE J. Quantum Electron. 8, 661-669 (1972).

10. S. Ura, S. Murata, Y. Awtsuji, and K. Kintaka, "Design of resonance grating coupler,” Opt. Express 16 (6), $12207-$ 12213 (2008).

11. A. Massaro, and T. Rozzi, "Rigorous time-domain analysis of dielectric optical waveguides using Hertzian potentials formulation," Opt. Express 14 (5), 2027-2036 (2006).

12. A. Taflove, S. C. Hagness, Computational Electrodynamic: the Finite-difference Time-domain Method, (Arthec House Publishers, sec. ed., London 2000), ch. 2,3,4,7.

13. G. Mur, "Absorbing boundary conditions for the finite-difference approximation of the time-domain electromagnetic field equations," IEEE Trans. Electromagn. Compat. 23, 377-382 (1981). 